\title{
A Bioinspired Approach to Tri-nor-Guaianes. Synthesis of (-)-Clavukerin A
}

Gonzalo Blay, Begoña Garcia, Eva Molina and José R. Pedro*

Departament de Quimica Orgánica, Facultat de Quìmica, Universitat de València, Dr.

Moliner 50, E-46100, València, Spain

SUPPORTING INFORMATION TABLE OF CONTENTS

${ }^{1} \mathrm{H}$ and ${ }^{13} \mathrm{C}$ NMR spectra of compound 6 p S-2

${ }^{1} \mathrm{H}$ and ${ }^{13} \mathrm{C}$ NMR spectra of compound 7 p S-3

Traces NOESY spectrum of compound 7

p S-4

${ }^{1} \mathrm{H}$ and ${ }^{13} \mathrm{C}$ NMR spectra of compound 8

p S-5

${ }^{1} \mathrm{H}$ and ${ }^{13} \mathrm{C}$ NMR spectra of compound 9 p S-6

${ }^{1} \mathrm{H}$ and ${ }^{13} \mathrm{C}$ NMR spectra of compound $\mathbf{1}$ p S-7 


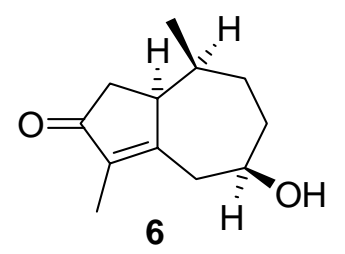

${ }^{1} \mathrm{H}$ NMR (400 MHz, $\mathrm{Cl}_{3} \mathrm{CD}$ )
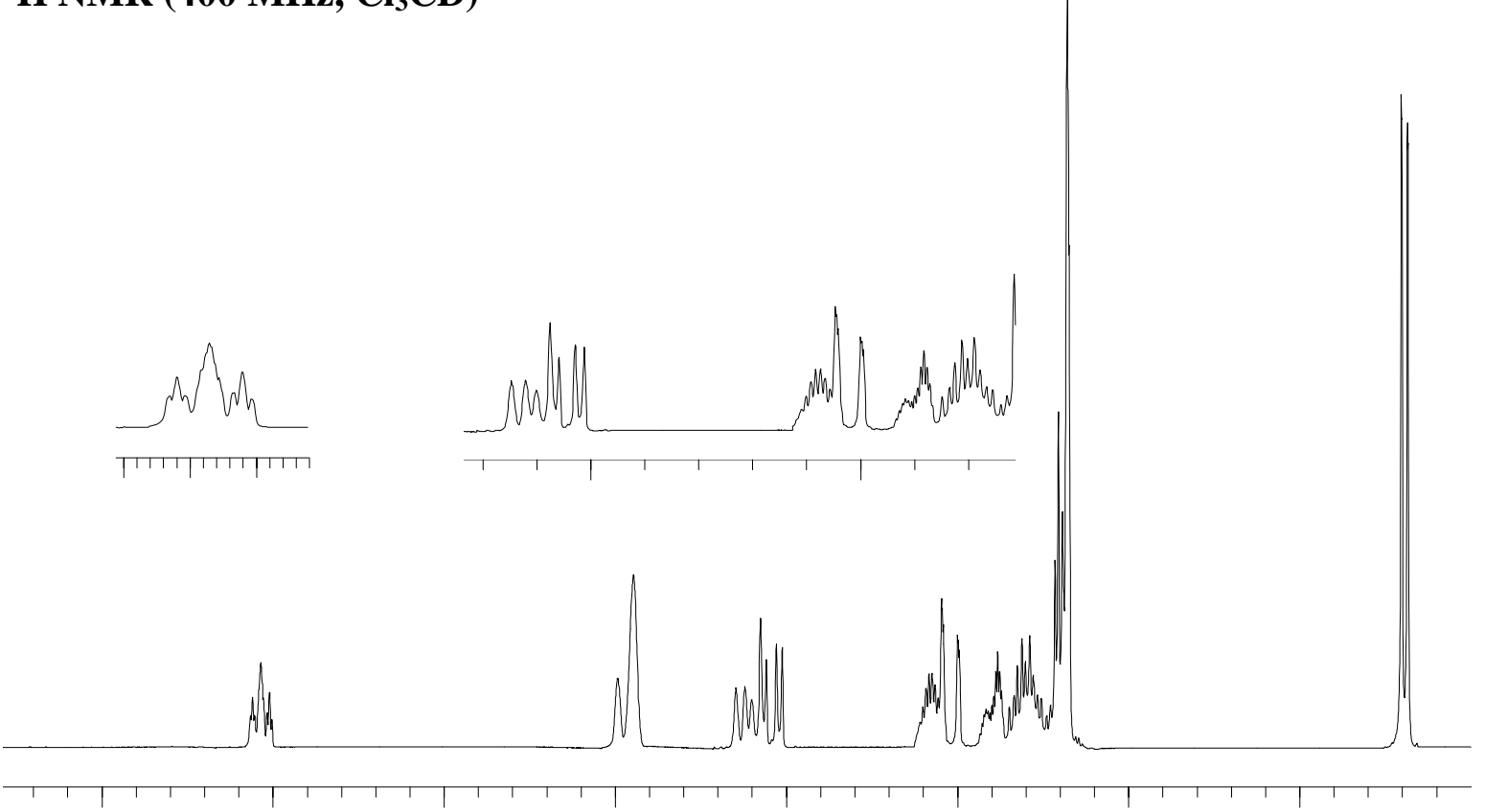

${ }^{13} \mathrm{C}$ NMR (75 MHz, $\left.\mathrm{Cl}_{3} \mathrm{CD}\right)$

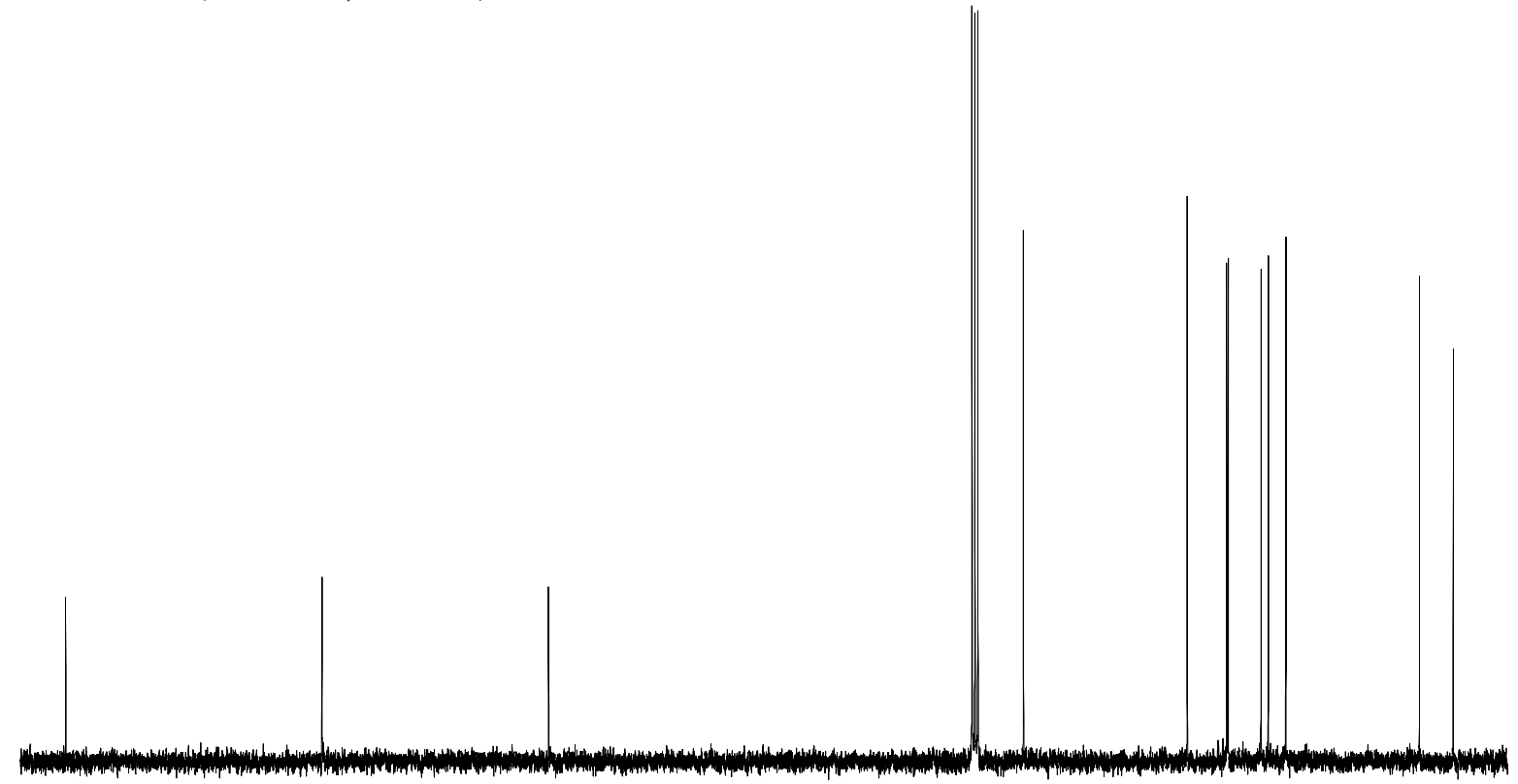




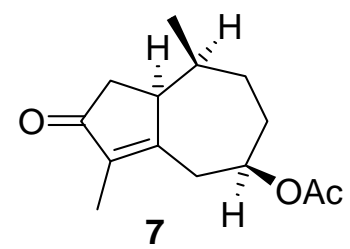

${ }^{1} \mathrm{H}$ NMR (300 MHz, $\left.\mathrm{Cl}_{3} \mathrm{CD}\right)$
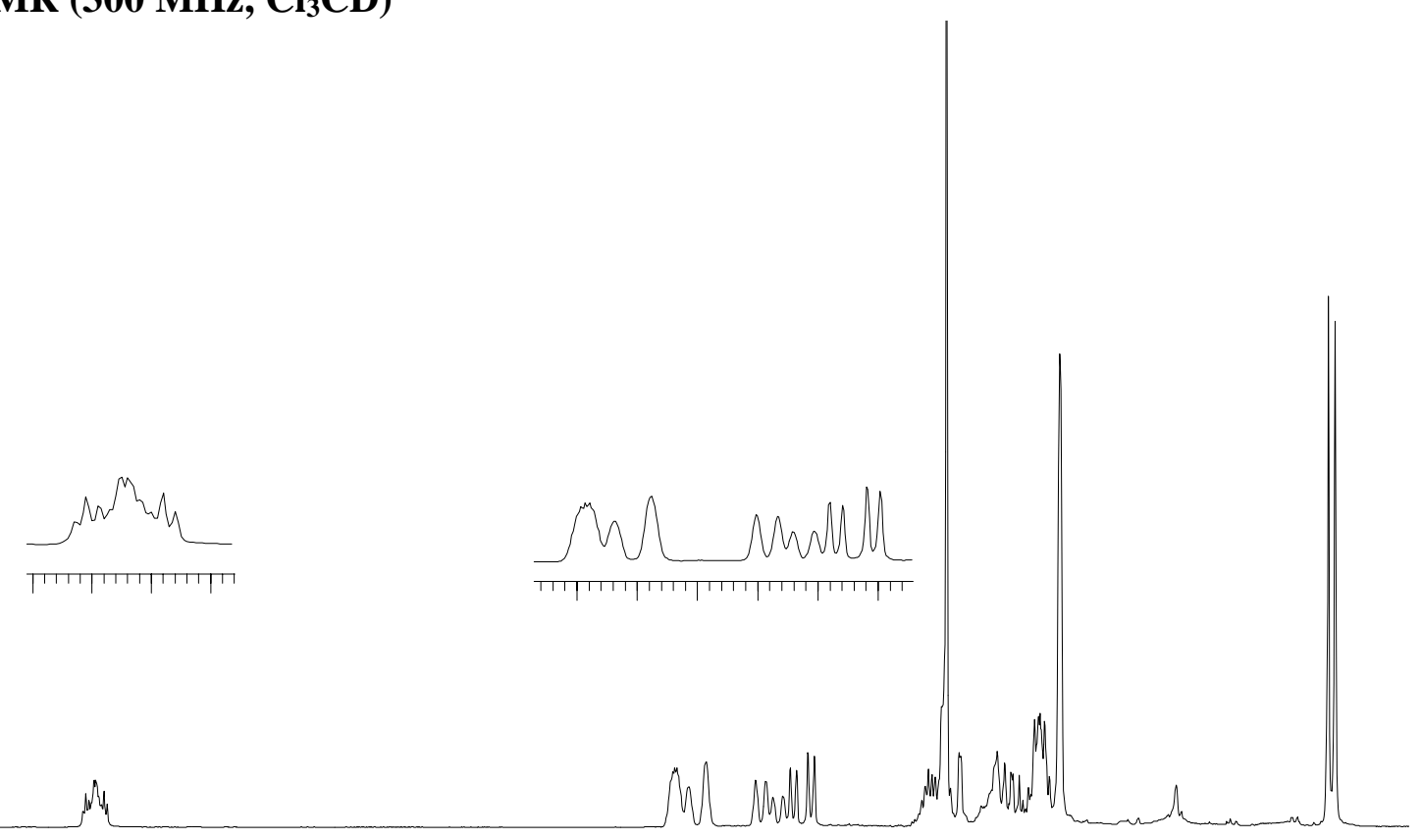

\section{${ }^{13} \mathrm{C}$ NMR $\left(75 \mathrm{MHz}, \mathrm{Cl}_{3} \mathrm{CD}\right)$}

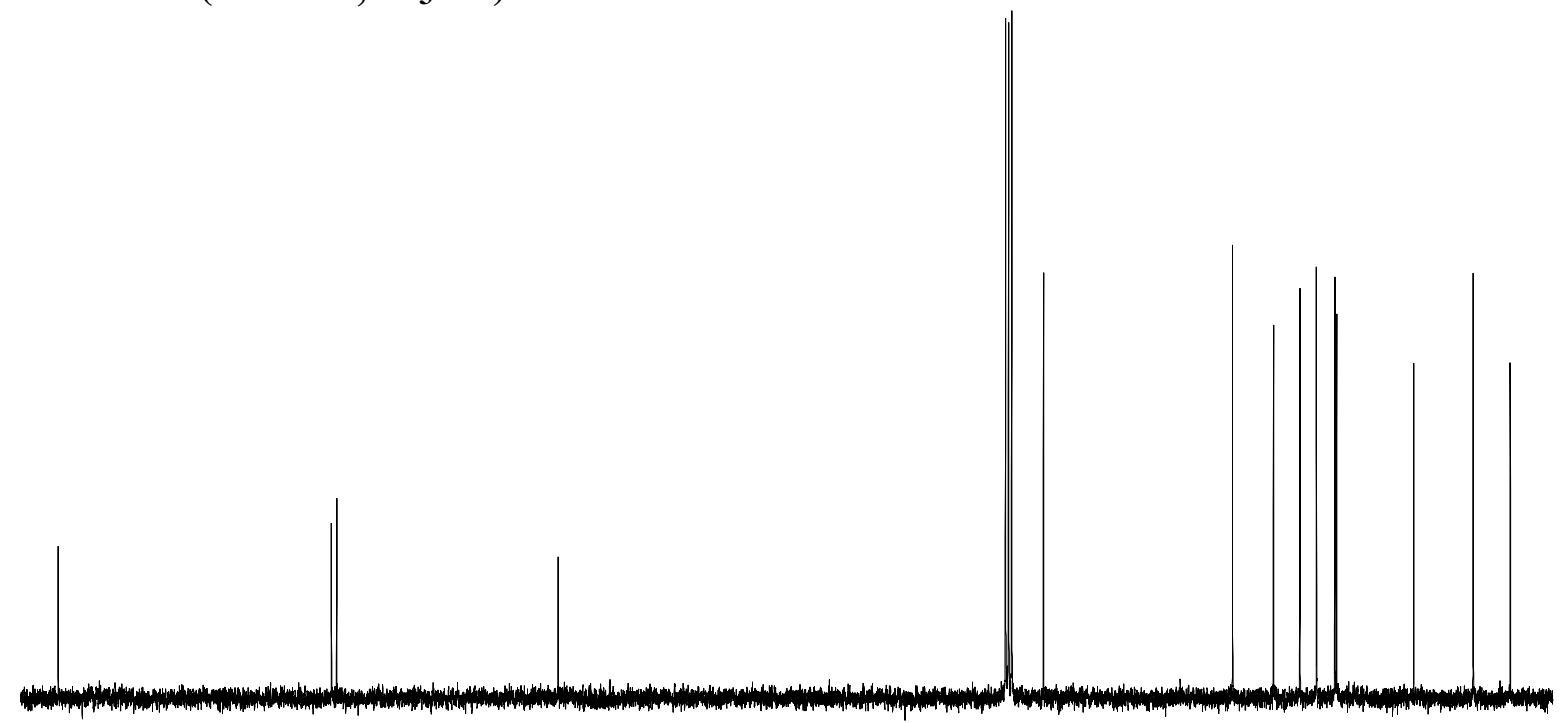




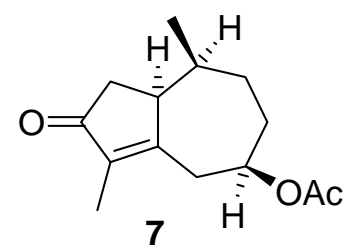

\section{NOESY (400 MHz, $\mathrm{Cl}_{3} \mathrm{CD}$ ): Traces}

ए0।

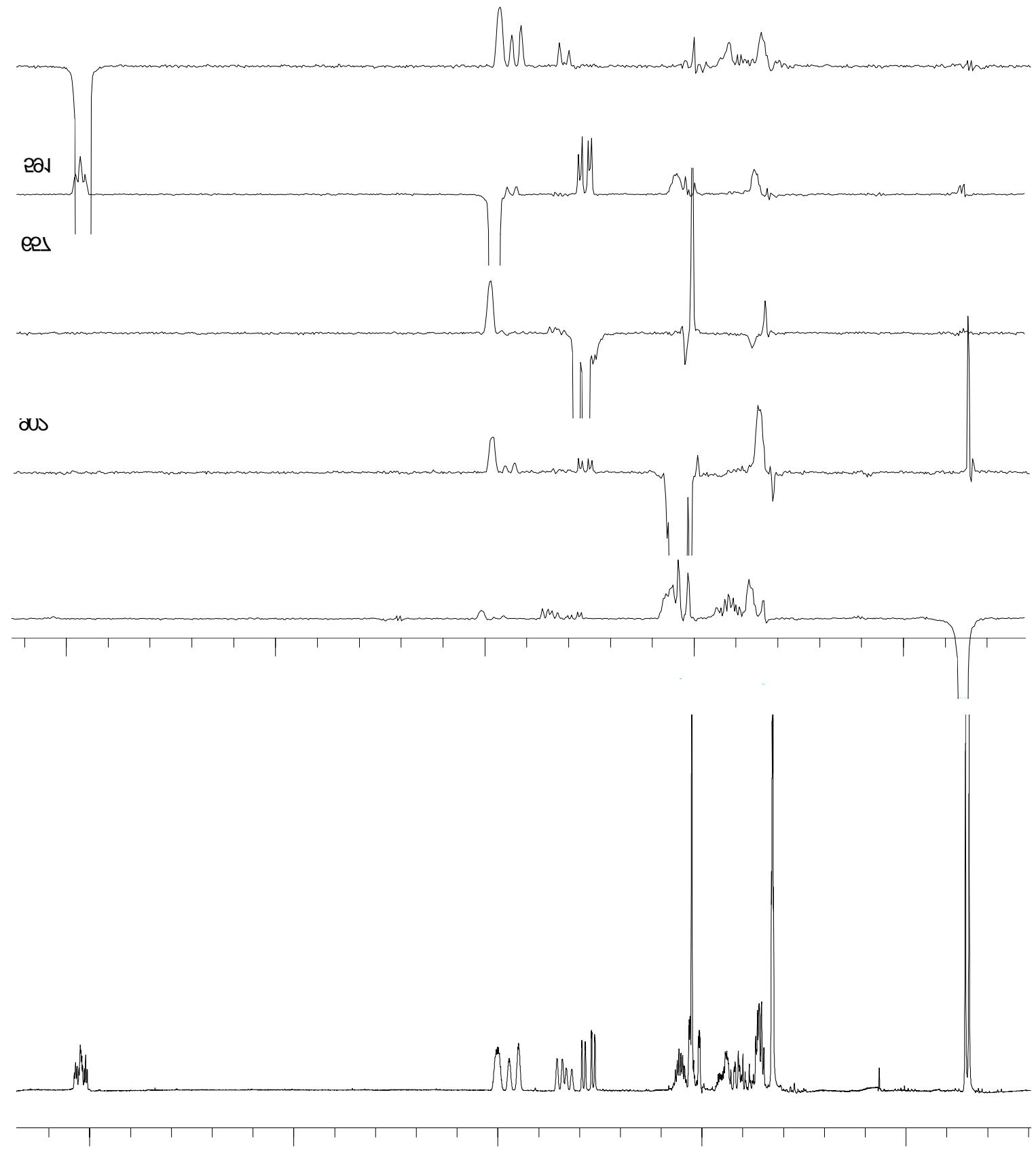




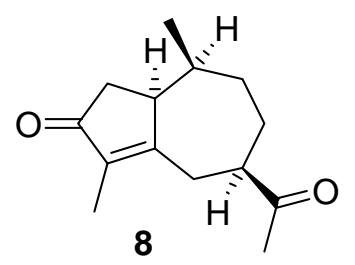

${ }^{1} \mathrm{H}$ NMR (300 MHz, $\left.\mathrm{Cl}_{3} \mathrm{CD}\right)$
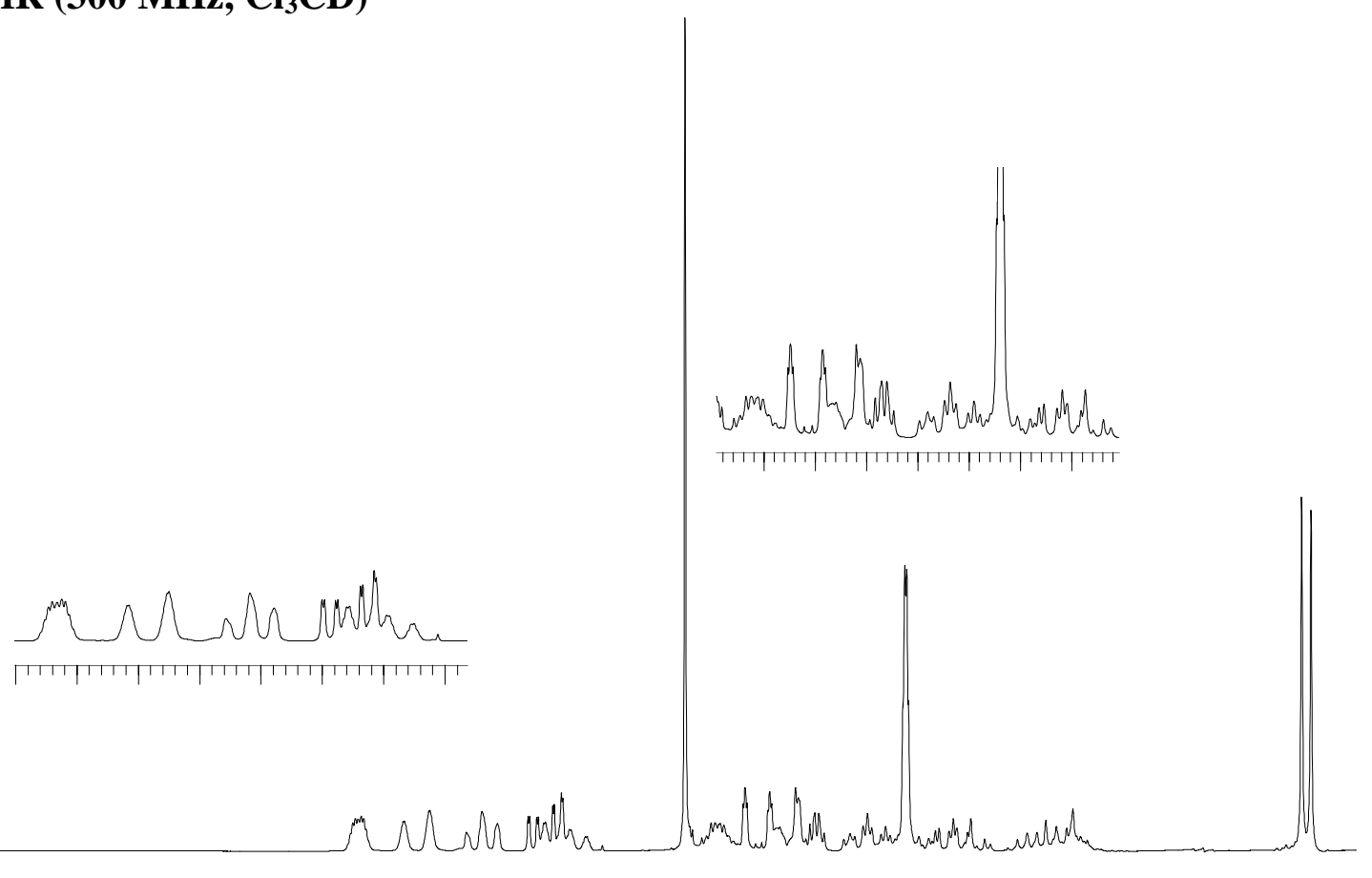

\section{${ }^{13} \mathrm{C}$ NMR (75 MHz, $\left.\mathrm{Cl}_{3} \mathrm{CD}\right)$}




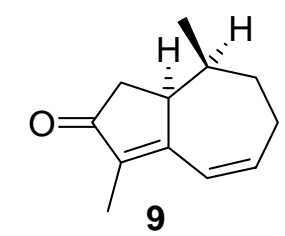

${ }^{1} \mathrm{H}$ NMR (400 MHz, $\mathrm{Cl}_{3} \mathrm{CD}$ )

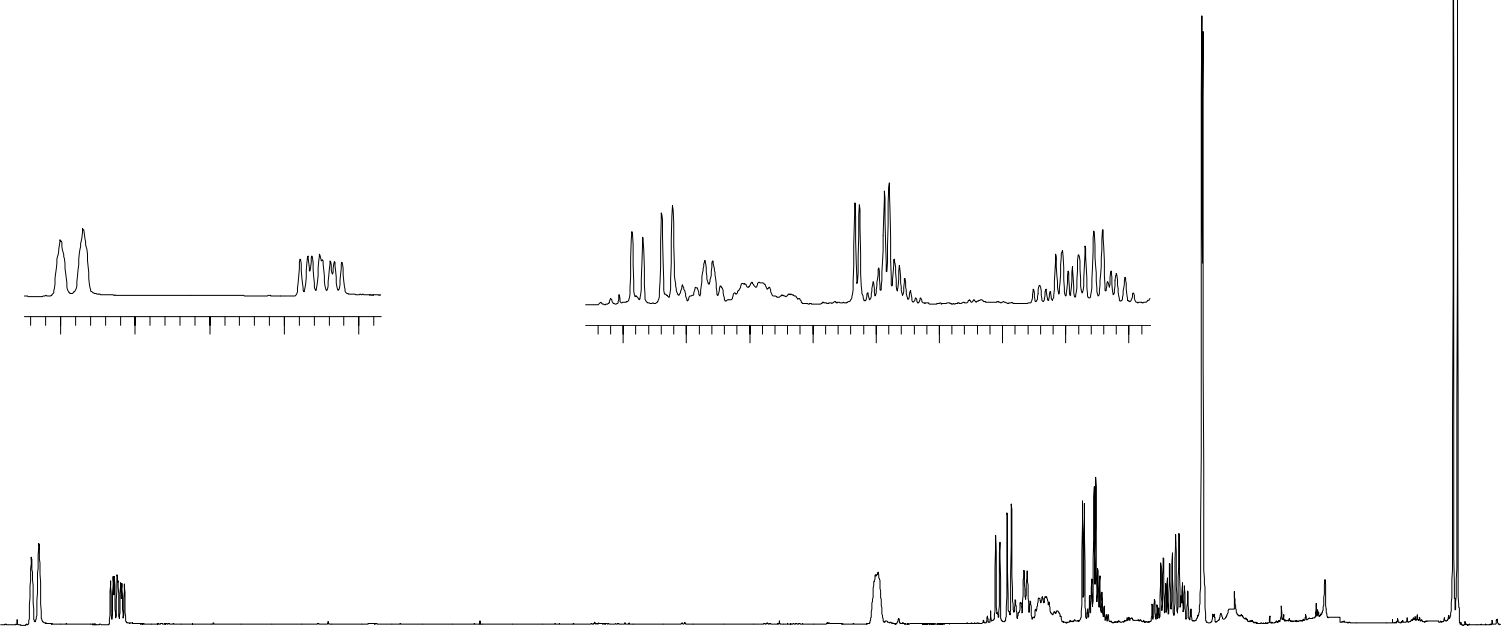

${ }^{13} \mathrm{C}$ NMR (75 MHz, $\mathrm{Cl}_{3} \mathrm{CD}$ ) 


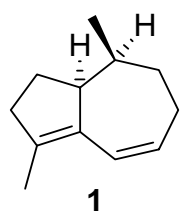

${ }^{1} \mathrm{H}$ NMR (400 MHz, $\left.\mathrm{Cl}_{3} \mathrm{CD}\right)$

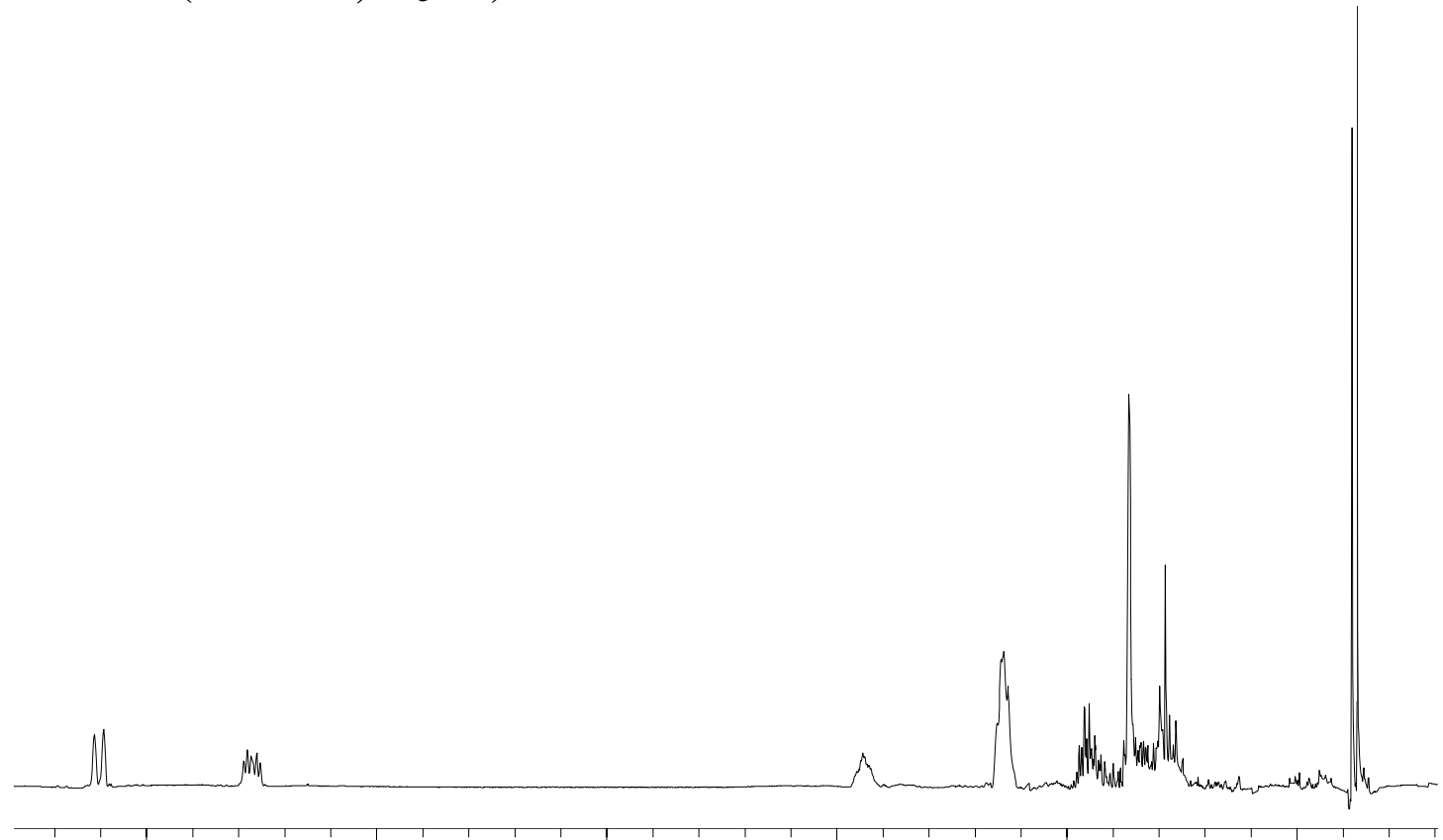

${ }^{13} \mathrm{C}$ NMR (75 MHz, $\left.\mathrm{Cl}_{3} \mathrm{CD}\right)$

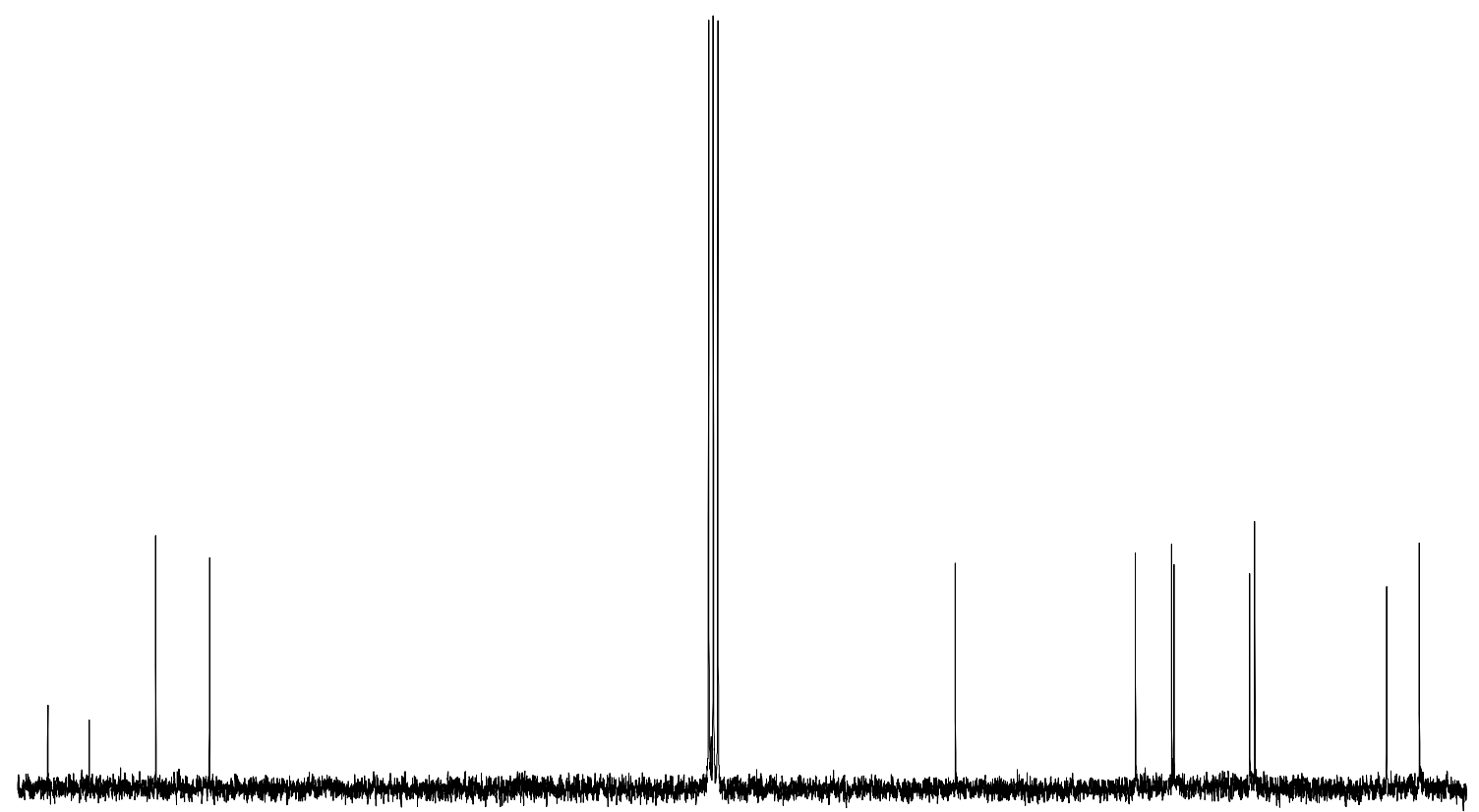

